\title{
Active and Attentive LADAR Scanning for Automatic Target Recognition
}

\author{
Michael T. Mamanakis, R. Rees Fullmer, Robert T. Pack and Scott Budge \\ Utah State Universtiy, 4170 Old Main Hill, Logan, UT 84322, USA
}

\begin{abstract}
In this work we examine the dynamic implications of active and attentive scanning for LADAR based automatic target/object recognition and show that a dynamically constrained, scanner based, ATR system's ability to identify objects in real-time is improved through attentive scanning. By actively and attentively scanning only salient regions of an image at the density required for recognition, the amount of time it takes to find a target object in a random scene is reduced. A LADAR scanner's attention is guided by identifying areas-of-interest using a visual saliency algorithm on electro-optical images of a scene to be scanned. Identified areas-of-interest are inspected in order of decreasing saliency by scanning the most salient area and saccading to the next most salient area until the object-of-interest is recognized. No ATR algorithms are used; instead, an object is considered to be recognized when a threshold density of pixels-on-target is reached.
\end{abstract}

Keywords: LADAR/LIDAR scanning, ATR/AOR, visual saliency, active scanning, attentive scanning, active perception, visual attention

\section{INTRODUCTION}

Since the industrial revolution, engineers have worked to allow machines to do tasks that are tedious or dangerous for humans. Many people take for granted the ingenuity that modern technological marvels embody; from what is seen on television any given evening, one might get the idea that with the help a computer assembling a part, finding a person in a crowd, identifying weapons in luggage or sending a robot to rove Mars are trivial tasks - just point-and-click. Few people marvel at their own ability to assemble a bicycle from a box of parts, find a friend in a crowd, categorize previously unseen objects using heuristics, or explore and interact with a foreign environment. Many people have thought, at some time or the other, "If only my computer could do this," or, "I wish I had a machine to do this for me." Such thoughts led the industrial revolution.

The rapid pace of technological development has given us computers and machines capable doing some of the amazing things that we take for granted. We are now undergoing an "autonomous" revolution wherein the real question is, "How do we do that which we do?" How do we find the right part in a box and then find the right hole to put it in? How do we even find the box in the first place? How do we find a person in a crowd or anywhere else? How do we make sense of new things we see and liken them to things we have seen at another time? How do we avoid obstacles when traversing new terrain? Though many of these tasks are thoughtless, natural automatic for people, the same is not true for machines. Much thought has been put into how people see and interact with an environment in order to allow machines to perform the same "simple" object recognition that people perform countless times each hour.

\subsection{Automatic Target/Object Recognition}

The field dedicated to the study of machine based object recognition is, depending on the application, referred to as automatic target recognition (ATR) or automatic object recognition (AOR) with ATR being used primarily for military applications AOR for civilian applications. Both fall into the broader fields computer vision (CV) and artificial intelligence (AI) and draw from the fields of image processing, image analysis, and pattern recognition. ${ }^{1}$

Further author information: (Send correspondence to R.R.F)

M.T.M: E-mail: michael.mamanakis@usu.edu

R.R.F.: E-mail: rrfullmer@engineering.usu.edu

R.T.P: E-mail: rtpack@cc.usu.edu

S.B.: E-mail: scott.budge@ece.usu.edu 


\subsubsection{Applications}

Some applications of AOR include: the shopping-cart problem, where a list of the cart's contents is desired without the use of bar codes; the bin-of-parts problem, where a certain part is sought; video surveillance, where the state of a space and the presence, or lack, of people or a particular person is of interest; image searches, where the desire is to find types of images, perhaps pictures with cows and bicycles, or any other object on the Internet or another image repository; traffic monitoring for better traffic management; medical image analysis to find tumors or cancerous growths; smart cars which can driver for, or assist, the driver in guiding the car; terrain aided navigation for pilots flying low to the ground, or astronauts trying to land on the Moon. ${ }^{2}$

Martial applications include: fire-and-forget and lock-on-after-launch missiles, air defense, assisted tank targeting, artillery targeting, mine detection, friend-or-foe identification, pilot assistance, high value target prioritization, and human analysis load reduction..$^{3,4}$

\subsubsection{Methods}

The "how" of AOR follows a typical pattern regardless of the actual algorithm used to perform recognition: preprocessing, detection, segmentation, feature computation, selection, classification, prioritization, tracking, and aim point selection. ${ }^{1}$ Borrowing from research on "how" human recognition (HR) is accomplished, ${ }^{5}$ a more general and applicable (to this paper) ATR flow can be defined: image acquisition, pre-attention, attention, and post-attention. This generalized ATR flow provides a better framework for evaluating non-traditional ATR approaches where image acquisition is not necessarily finished when image processing begins.

The image acquisition phase of the AOR cycle is critical for two reasons: first, accuracy of recognition is directly related to the image resolution and quality, ${ }^{1,6,7}$ i.e., any ATR algorithm can only provide good, reliable recognition with good, reliable data; second, image acquisition is not always instantaneous and can be slow depending on the sensor used. ${ }^{8}$ Fine resolution imaging sensors have been developed, which can achieve high quality discrimination; unfortunately, the relative slowness associated with such systems can sometimes prevent their use in real-time systems.

The actual computation and processing that takes place in the pre-attention, attention and post-attention steps of the ATR cycle vary depending upon the ATR approach used. Pre-attentive process include any means whereby the attention of the system is drawn. Pre-attention processes can be run in parallel when several sensor modalities are used as on a multi-sensor platform. This is the phase where parts of an image, or the environment, are selected for further scrutiny. Attentive process provide the further scrutiny cued by the preattentive processes. Attention is where cognizance and recognition occur and may involve gathering more images and data to improve the quality of recognition. Post-attentive processes can be grouped into two broad categories based on the conclusions of attentive processes: object recognized or object not recognized. Depending on the ATR/AOR application, actions for "object recognized" might include charging a customer for the contents of his shopping-cart, notifying emergency services that an accident has occur, or changing from search to attack mode in an autonomous cruise missile. Actions for "object not recognized" conclusion include, among other things, keep looking, look somewhere else, or take a closer look at everything.

\subsubsection{Sensors}

Several types of sensors are used to collect images for AOR processing including: FLIR, RADAR, SAR, 2D visible EO, and 3D LADAR. Of these sensors, LADAR based AOR, in particular, has been shown to be very effective and to have low false alarm rates. ${ }^{9,10}$

LADAR scanners collect intensity information and, as the acronym implies, range and direction information. The collected data can be used to create what are known as range and intensity images. A technical report on the performance of next-generation is available from NIST. ${ }^{11}$ This freely available technical report is an excellent primmer on LADAR scanners for the interested reader.

LADAR scanners provide quality information for ATR/AOR. The combination of small pixel size and fine range resolution available with LADAR scanners, provide better information for processing than other 3D range sensors, such as synthetic aperture RADAR. This higher quality information directly results in better AOR performance. 


\subsubsection{Performance}

Real-time, LADAR-based AOR effectiveness comes with the high cost of custom scanners. Custom scanners are developed where space is a premium and when scanning agility is required. Unlike AOR systems which use visual/infrared cameras to examine the field of regard, single beam LADAR scanners are constrained by the dynamics of the scanning mirror; unfortunately, the "fast" steering mirrors used in such systems can take several seconds to complete a raster scan at high enough resolution to identify objects. This is unacceptable in real-time situations where the object-of-interest (OI) may only be briefly available to scan due to object or platform motion.

\subsection{Visual attention}

Increasing sensor quality and resolution brings increasing data loads. A common approach to the problem of ATR complexity is to bring to bear the power of parallel processing; however, parallel processing is not a solution to the problem of complexity, rather, it is a means of reducing the waiting time for an answer based on collected data. A better solution is to process the data in an intelligent fashion. ${ }^{12,13}$ A better solution involves reducing the amount of data gathered and processed.

\subsubsection{Foveation}

One of the first HR strategies used to "pay visual attention" is foveation of the retina. The human eye does not have an even distribution of resolution due to the placement of cones and rods on the retina. The fovea has a higher concentration of the smaller cones, thus higher viewing resolution. The parafovea and perifovea regions surrounding the fovea have more of the larger, less densely packed, rods.

Lower resolution peripheral regions allow awareness without the brain processing larges amounts of information and can be thought of as a mechanical limit on information intake. Foveation is merely a biological embodiment of the "pay attention where it counts" principle and has lent itself to many uses ${ }^{14,15}$ including ATR/AOR. McKee et al state: "Foveal ATR exploits the difference between detection and recognition resolution requirements and sacrifices peripheral acuity to achieve a wider FOV, giving faster search with greater resolution where needed ". ${ }^{16}$ This suggest that given an agile, steerable LADAR scanner, AOR performance would improve by varying the density of the scan such that greater resolution is applied where it is needed and not elsewhere.

\subsubsection{Visual saccade}

Increased resolution, i.e., the fovea, is brought to bear on an region-of-interest (ROI) in the field-of-regard (FOR) when a pre-attentive mechanism activates a visual saccade, or jump of the eye from one position to the next. Under scrutiny, following a saccade, a ROI becomes the focus-of-attention (FOA). Saccading allows the use of lower-resolution parafovia and perifovia to dramatically increase the size of the FOR without affecting ability to discern and discriminate.

Many AOR variants based on foviation and saccading, including foveated FPA, or zoom lenses with uniform FPA used to achieve a foveation-like effect, have been proposed. ${ }^{17-23}$ It is important to note that most prior research has simulated saccading by extracting portions of a full-FOV frame for processing by an AOR algorithm. Likewise, zooming has been simulated by physically moving the sensor or down sampling from the initial capture resolution. ${ }^{24,25}$ Though image extraction for processing has been shown to be effective at reducing computation time to varying levels and to be useful for AOR, ${ }^{2}, 16,17,22,23,25-34$ ignoring the image acquisition time, especially LADAR acquisition time, is ignoring an important, and sometimes lengthy, part of the real-time AOR process.

\subsubsection{Saliency}

The question still remains, "What is it that makes a ROI interesting or salient?" Visual saliency is a "bottom-up, stimulus-driven signal that announces 'this location is sufficiently different from its surroundings to be worthy of your attention"' 35 or, in other words, a part of a image is salient if that part of the picture differs more from its surroundings than any other region of the image does its own surroundings.

Saliency activation is thought to be the mechanism whereby the attention of the eye is drawn to different regions of an image. ${ }^{36}$ This implies that saliency would be a good candidate for the "pre-attention" phase of the ATR process with a saliency map being used "to represent the conspicuity or saliency at every location in the 
visual field by a scalar quantity and guide the selection of attended locations based on the spatial distribution of salience". ${ }^{27}$ This concept has been investigated by Walther et al. ${ }^{32}$ and Rutishauser et al., ${ }^{30}$ among others.

\subsubsection{Top-down vs. bottom-Up}

The pre-attentive mechanisms which help determine saliency are thought to bring attention to salient regions of an image though two mechanisms: top-down attention and bottom-up attention.

Bottom-up attention is thought to be driven by images features such as orientation, intensity, color, etc. ${ }^{5,27}$ Bottom-up cues can been seen readily through what is known as pop-out; for instance, a red ball in a bucket of green balls, a vertical line in a field of horizontal lines, or a square in circles are examples of pop-out based on unique color, orientation, respectively. Bottom-up attention is a parallel process over the many feature channels considered.

Top-down attention, in one form, can be thought of as a biasing on the relative effect of bottom-up cues. This guidance of the search process is less well understood than the bottom-up process. One way to implement top-down biasing in search is to train for cues as in. ${ }^{2}$ An example of top-down biasing is to more heavily weight pertinent feature maps when combining those feature maps to form the saliency map; e.g., when searching for a note written on a small, orange paper in a clutter of papers of various sizes and colors, the OI would be found more quickly if the feature maps were biased toward orange in the small scale. In this paper clutter there may only be one, thus unique, green note, but it will be ignored because orange is the color of interest. Top-down gives priority in visual search ${ }^{37}$ and is a strong factor in guided visual search. ${ }^{5}$

\subsection{5 iNVT}

The iLab Neuromorphic Vision C++ Toolkit (iNVT) was initially developed by Itti, Koch, and Niebur ${ }^{27,35}$ and based on earlier work of Koch and Ullman. ${ }^{36}$ iNVT works to create saliency maps in the following manner. Different scales are computed using Gaussian pyramids allowing for feature comparison across different scales. Three types of features are examined for stationary images: color, intensity, and orientation. Color and intensity feature maps are created by examining the image in a center-surround fashion, where pixels are compared to their surrounding to give context, and placing them in Gaussian pyramids. Orientation feature maps are created by computing oriented Gabor pyramids. Gabor filters are the product of a cosine grating and 2D Gaussian envelope. Gabor pyramids are formed when Gabor filters are applied across several scales. ${ }^{27}$ These maps are combined into feature maps and then into conspicuity maps using a weighting system which promotes maps in which there are fewer peaks. The original implementation of iNVT combined 42 feature maps into the saliency map.

\subsubsection{Performance and validity of saliency models}

Once attention is activated, the biological response is to saccade and inspect. The biological response suggests that the equivalent electro-mechanical sensor response should be to saccade and inspect.

Experiments using visual saliency models have been performed by Ouerhani et al. ${ }^{38}$ and Parkhursta et al. ${ }^{31}$ to validate the model of Koch and Ullman ${ }^{36}$ and other similar models. A strong correlation between HR ROI and CV selected ROI was shown when a new scene was presented to a human viewer. Human fixations were determined by tracking eye movement and fixation. As viewing time elapsed, the correlation between computer and human ROI decreased. This decrease is thought to be caused by a shift from bottom-up to top-down queuing.

Saliency works well in guiding attention for further processing. Bonaituo and Itti wrote that, "Attentionguided recognition with object prioritization drastically reduces the average number of key-point comparisons and the average matching duration". ${ }^{39}$ Rutishauser et al. wrote, "pure bottom-up attention can extract useful information about the location, size and shape of objects from images". ${ }^{30}$ 


\subsection{Saliency and object recognition}

An essential distinction must be made: saliency is not object recognition. The degree to which an area of an image is "interesting" in the sense of saliency does not, and can not, give any indication as to what is in that area of the image. ${ }^{30}$ This does not mean that saliency is not useful for object recognition or that no correlation exists between the saliency map and the location of the OI; saliency driven "bottom-up attention is indeed useful for a variety of applications". ${ }^{30}$

Using bottom-up saliency algorithms to select ROI for further computational interrogation by extraction from full-FOV images has been shown to be effective at reducing the number of ROI examined before the OI is found. The reduction of examined FOR translates directly to computationally faster AOR time-to-recognition (TTR). This reduction in FOR examined, and thus TTR, is even greater when top-down training is implemented. ${ }^{2,34}$

\subsection{Contribution}

As systems limited by computational power can benefit from saliency driven object recognition, so can system limited by dynamic constraints. What follows is a brief outline of a study on the benefits that can be realized by using saliency to guide actively and attentively guide a LADAR scanner for ATR/AOR purposes. By actively scanning only salient regions of an image at the density required for recognition, the amount of time it takes to find a target object in a random scene is reduced.

\section{METHODOLOGY}

\subsection{Scope}

The combination of EO visual cameras for saliency mapping and saccade direction with LADAR for high-fidelity object recognition was examined. Proof of concept example were investigated followed by several Monte Carlo simulations.

\subsection{Assumptions}

The scanner consists of a single scan mirror, a single guidance mirror and a single LADAR beam with the following characteristics: The LADAR laser has a 500-meter minimum range and 5,000-meter maximum range. The beam divergence of the laser is sufficiently small to place the desired number of pixels-on-target (POT) at maximum range without pixels overlapping. The scan mirror has a four-degree radius circular range of motion, which, because rectangular raster patterns are scanned, gives a circumscribed raster square $5.67^{\circ}$ on edge. The guidance mirror has a $45^{\circ}$ radius circular range of motion and a $63.64^{\circ}$ on edge raster square. No object larger than the scan mirror FOV was scanned. The scanning mirror has a maximum acceleration of $1,000 \mathrm{rad} / \mathrm{s}^{2}$ and a maximum velocity of $10 \mathrm{rad} / \mathrm{s}$. The maximum velocity is based on maximum acceleration to mid-motion range followed by maximum deceleration from stop to stop with zero velocity boundary condition. The scanner platform is stationary as is the OI. The physical size of the OI is known. Following a saccade, once the LADAR beam is in proximity of the OI, knowledge of OI size allows the FOA bounding box to be intelligently sized. Physical size and range allow the post-spacing (PS) to be adaptively set while scanning in order to place the desired number of pixels in the FOA and thus, eventually, the target.n The saliency algorithm will not be trained to provide any top-down guidance. With the lack of top-down guidance, no assumption can be made about the relationship of the OI's saliency to other object's saliences, thus no assumption can be made about the best saccade order. No individual AOR or ATR algorithm was used in evaluating the hypothesis. ATR was be simulated by pixels-on-target (POT), whereby recognition occurs when a minimum number of POT is attained. ${ }^{6}$ The recognition threshold was set at 500 POT. It is assumed that sufficient computational power is available such that the time required to compute saliency maps and run ATR algorithms is small compared to raster scan time. 


\begin{tabular}{lll} 
& \multicolumn{2}{c}{ Table 1. System Parameters } \\
Variable & Description & Base Case \\
\hline $\mathrm{u}$ & Azimuth positions the ROI & full-FOV \\
$\mathrm{v}$ & Elevation positions the ROI & full-FOV \\
$\mathrm{N}$ & The number of ROI & random in range \\
$N_{\min }$ & Minimum number of ROIs & 1 \\
$N_{\max }$ & Maximum number of ROIs & 10 \\
$\mathrm{r}$ & Range to the ROI & random in range $500 \mathrm{~m}-5 \mathrm{~km}$ \\
$r_{\min }$ & Minimum range to ROI & 500 meters \\
$r_{\max }$ & Maximum range to ROI & 5000 meters \\
$\alpha$ & Width of the OI, FOR, or FOA & 10 meters \\
$\beta$ & Height of the OI, FOR, or FOA & 3 meters \\
$P_{r}$ & ATR POT recognition threshold & 500 \\
$P_{d}$ & ATR POT detection threshold & $P_{r} / 8$ \\
$a_{\max }$ & Scanner maximum acceleration & $1000 \mathrm{rad} / \mathrm{s}^{2}$ \\
$v_{\max }$ & Scanner maximum velocity & $10 \mathrm{rad} / \mathrm{s}$
\end{tabular}

\subsection{Metrics}

Improvement in performance is measured as the ratio of the baseline scan time to the active scanning TTR and called the performacne factor (PF). The baseline scan is a full-FOV scan at the ATR recognition threshold. Performance factors greater than one indicate enhanced performance; factors equal to one indicate par performance; factors less than one indicate decreased performance. For example, if the baseline scan requires three seconds and attentive scanning requires one second then the scan time performance factor, $P F$, will be three, indicating a $3 \mathrm{x}$ improvement in time to recognition.

\subsection{Proof of concept procedure}

A small number of scenes were created using in LadarSIM ${ }^{\mathrm{TM}}$, a scene-based LADAR simulator. Each scene consisted of a randomly placed tank in randomly placed trees. For each scene, the saliency software was used to generate the saliency map and saccade queue. The saliency map was converted to appropriately size ROI. Post spacings were calculated based on the size of each ROI, which is dependent on the ROI's range.

Once all the scan parameters were calculated, ROIs were programed into LadarSIM ${ }^{\mathrm{TM}}$ as a sequence of scans in the order given by the saliency software. LadarSIM ${ }^{\mathrm{TM}}$ processed the scan queue and returned a set of range and intensity images. Next, the full-FOR scan was programed into LadarSIM ${ }^{\mathrm{TM}}$ using a PS based on the ATR POT threshold for an OI at maximum LADAR range and processed giving another scan time and set of range and intensity images.

\subsection{Monte Carlo procedure}

Scripts and functions were written to simulate the active and attentive LADAR scanning process as explained for the proof of concept examples. A Monte Carlo simulation was run and the data from the simulation was used to calculate the performance factors. Many variations of the system parameters listed Table 1 were examined. A summary of the observed trends is given in the following section.

\section{RESULTS}

\subsection{Proof of Concept Example}

The proof of concept scene scenario is described in Table 2(a). The active scan performance statistics are tabulated in Table 2(b). Figure 1 shows the saliency flow for example scene one including the EO image from the scanners point of view as well as the associated conspicuity and saliency maps and the derived saccade path. These scans were combined using the CAIL LADAR Viewer and are shown with the initial visual image and the ATR detection threshold scan in Figure 2. 


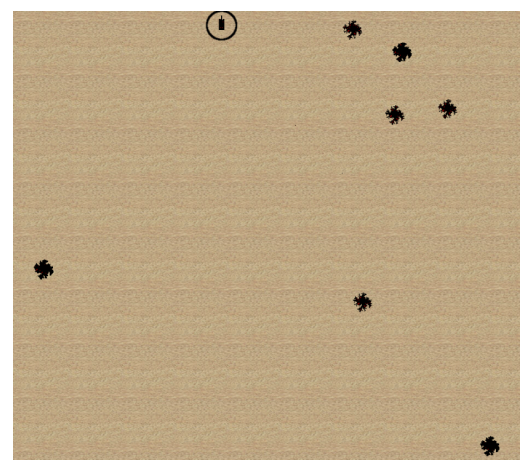

(a) EO image with tank circled.

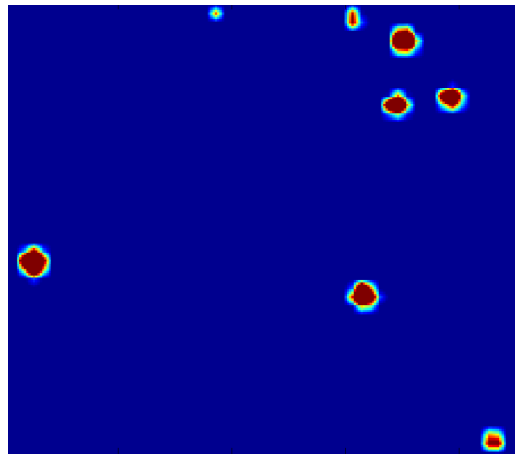

(b) Color conspicuity map.

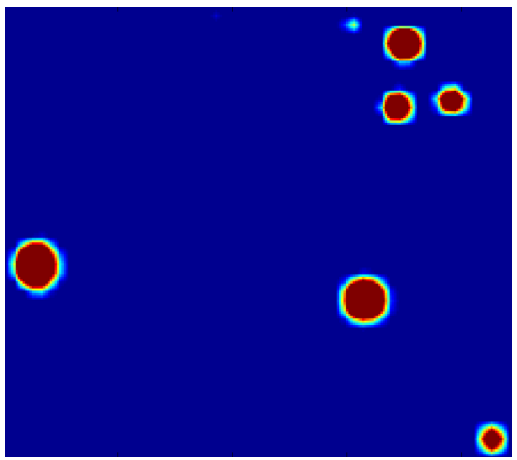

(d) Orientation conspicuity map.

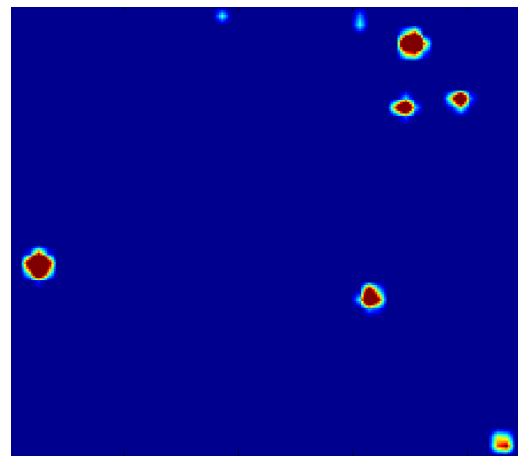

(c) Intensity conspicuity map.

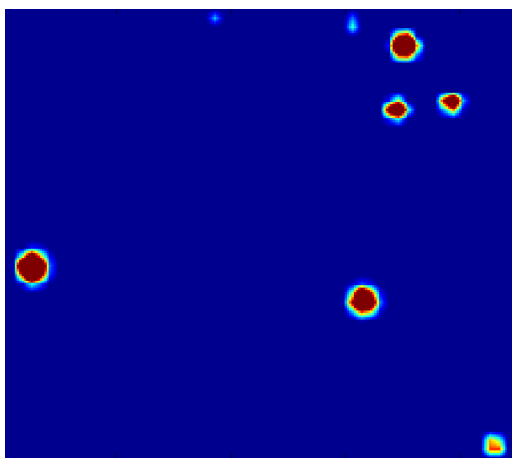

(e) Saliency Map.

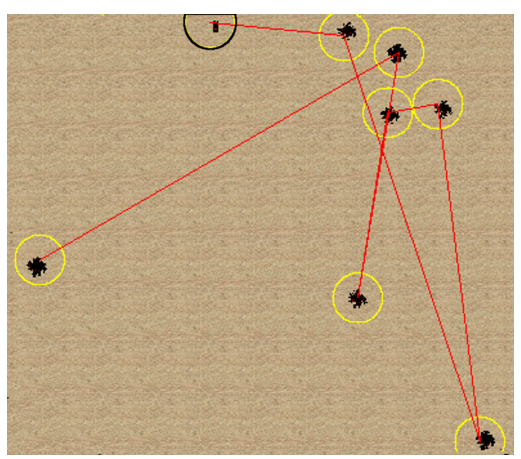

(f) Saccade Path.

Figure 1. Example scene: saliency flow. 


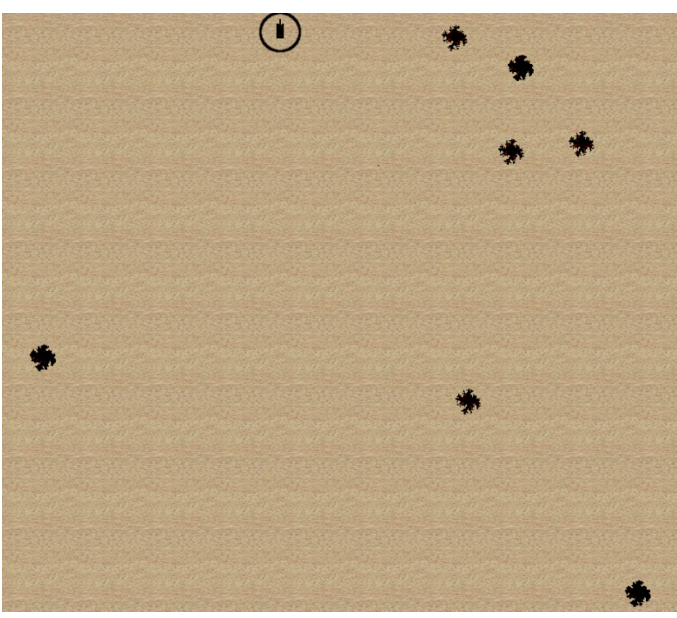

(a) EO image.

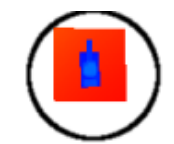

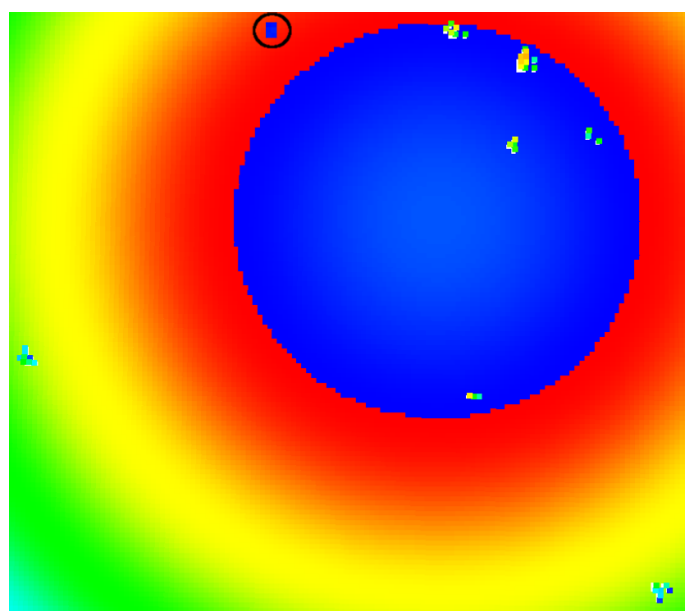

(b) Detection LADAR scan image.
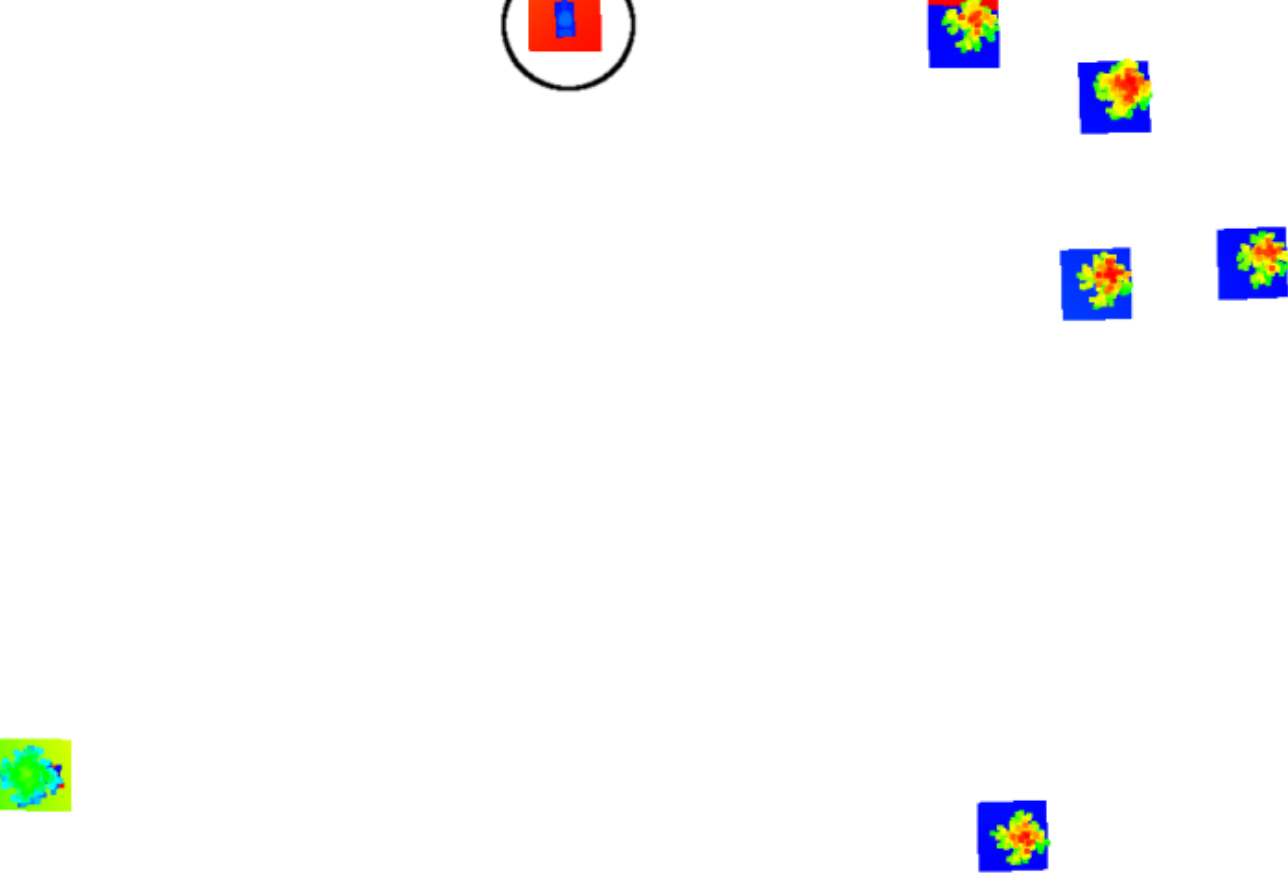

(c) Attentive LADAR scan image.

Figure 2. Example scene: Comparison of cropped EO (a), detection LADAR (b) and attentive LADAR (c) images. 
Table 2. Proof of Concept Example

(a) Scenario

\begin{tabular}{ll} 
Parameter & Value \\
\hline Approximate range & 3456 meters \\
Regions-of-interest & $8(7$ trees \& 1 tank) \\
$(\mathrm{x}, \mathrm{y})$ tree locations (in meters) & $(93,9),(-67,-202),(40,74),(-185,103)$ \\
& $(-89,16),(78,43),(36,38)$ \\
$(\mathrm{x}, \mathrm{y})$ tank location (in meters) & $(93,-80)$ \\
Raster grid size & 71 rows X 71 columns \\
Post spacing & 61 microradians
\end{tabular}

(b) Performance Statistics

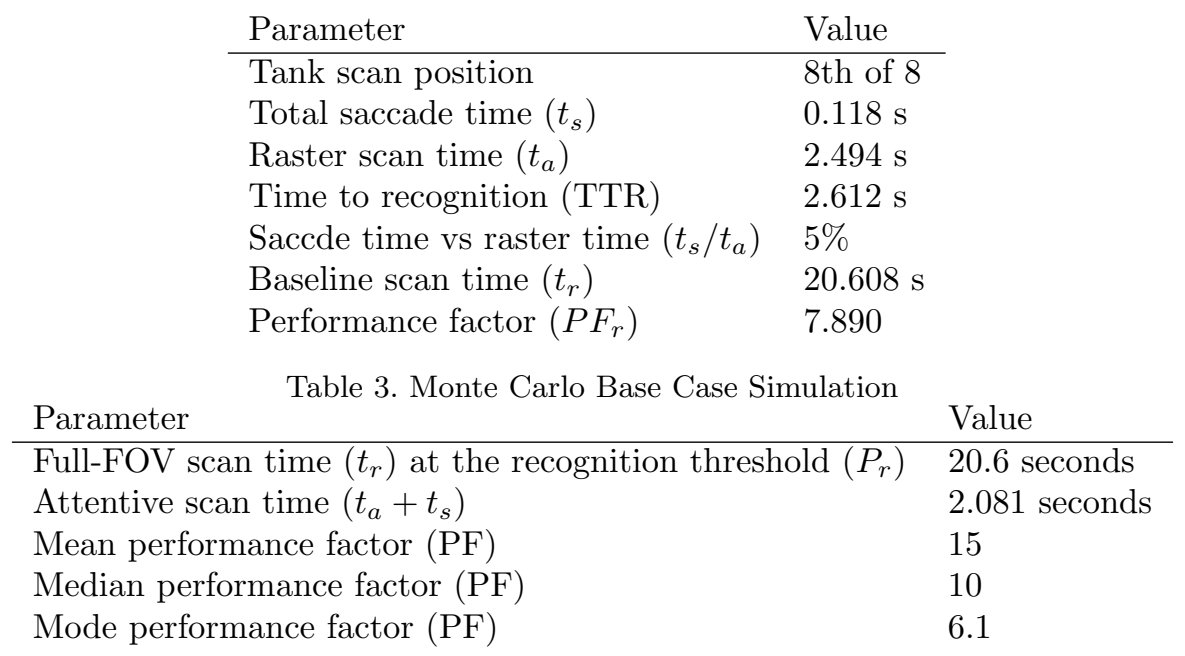

\subsection{Monte Carlo Simulation}

The Monte Carlo simulation presented is of the base case as listed in Table 1. The results of this simulation over 100,000 random scenes are shown in Figure 3. The results of this simulation are listed in Table 3.

\subsection{Performance Factor Trends}

The Monte Carlo simulation was used to examine how mean performance factor varied with changes in systems/scanner variables. The following trends were observed:

- Performance factor $(\mathrm{PF})$ increases with average range.

- PF decreases with increasing numbers of AOIs.

- PF decreases with increasing ATR/AOR thresholds.

- PF increases with increasing FOV widths.

- PF increases with maximum scanning mirror acceleration.

- PF decreases wit maximum scanning mirror velocity.

\section{CONCLUSION}

A dynamically constrained, scanner-based, ATR system's ability to identify objects in real-time is improved through attentive scanning. By actively and attentively scanning only salient regions of an image at the density required for recognition, the amount of time it takes to find a target object in a random scene was reduced. 


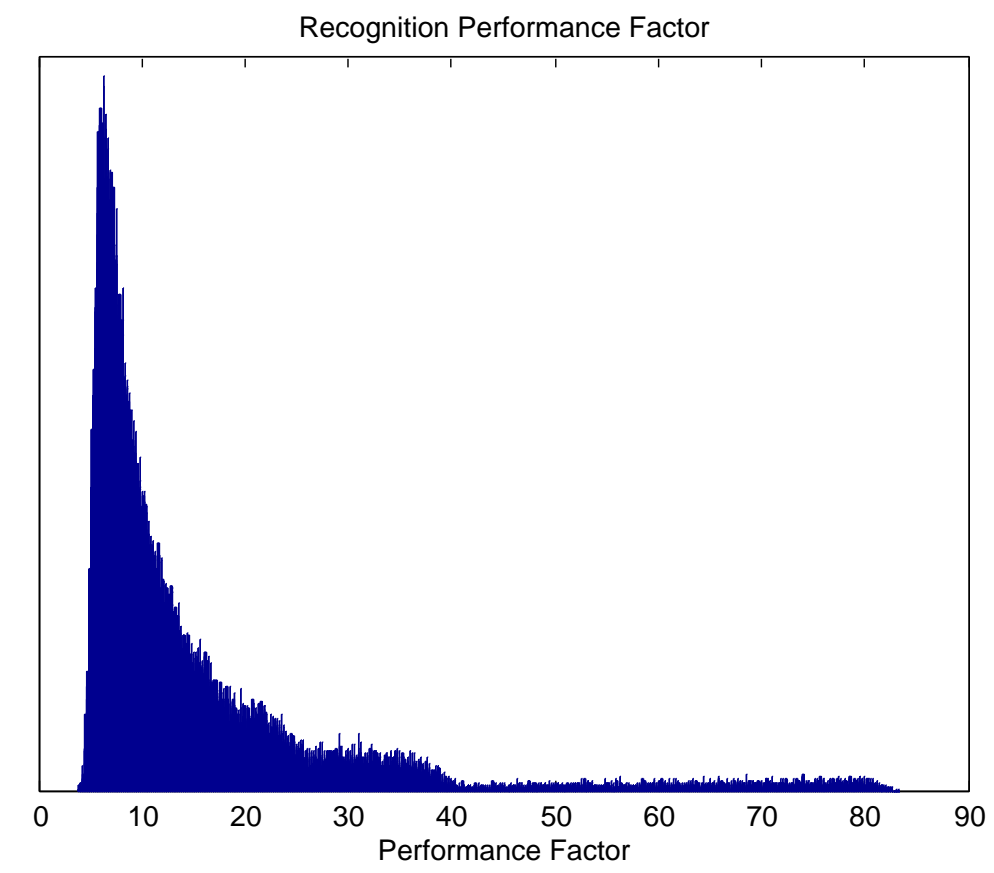

Figure 3. Histograms of the performance factor $(P F)$ for the hypothesis criterion base case.

The LADAR scanner's attention was guided by identifying areas-of-interest using a visual saliency algorithm on electro-optical images of a scene to be scanned. Identified areas-of-interest were inspected in order of decreasing saliency by scanning the most salient AOI and saccading to the next most salient AOI until the object-ofinterest, in this thesis a tank amidst trees, was recognized. No ATR algorithms were used; instead, an object was considered to be recognized when a threshold density of pixels-on-target was reached.

The described method had not yet been implemented in hardware; instead, LadarSIM ${ }^{\mathrm{TM}}$, a scene-based LADAR simulator, was used to examine a few proof-of-concept examples after which a Monte Carlo simulation was used to draw general statistical conclusions about this approach's efficacy. The amount of time required to find the objects-of-interest was compared to a baseline scan time wherein the full-field-of-view was scanned at recognition pixel densities. Performance of the scanning system was considered improved if the ratio of the baseline scan to the attentive scan, known as the performance factor, was greater than unity. Active and attentive scanning using saliency guidance showed improved performance with mean a performance factors of 15 .

\section{ACKNOWLEDGMENTS}

We thank the Koch Lab and Dirk B. Walther at the California Institute of Technology for use of the Saliency Toolbox and the iLAB and Laurent Itti at the University of Southern California for use of the iLab Neuromorphic Vision C++ Toolkit (iNVT) and associated figures.

\section{REFERENCES}

1. B. Bhanu, "Automatic target recognition: State of the art survey," IEEE Transactions on Aerospace and Electronic Systems AES-22, pp. 364-379, July 1986.

2. S. Frintrop, VOCUS: A Visual Attention System for Object Detection and Goal-directed Search. PhD thesis, Rheinische Friedrich-Wilhelms-Universität Bonn Institut Für Informatick and Fraunhoffer Institut Für Autonome Intelligente Systeme, 2006.

3. B. Bhanu and T. L. Jones, "Image understanding research for automatic target recognition," Aerospace and Electronic Systems Magazine, IEEE 8, pp. 15-23, Oct. 1993. 
4. J. E. Hilger, "Adaptive computing technology: An enabling processing technology for advanced sensor systems," IEEE Transactions on Systems, Man and Cybernetics 3, pp. 2178-2183, 1998.

5. J. M. Wolfe, "Guided search 2.0: A revised model of visual search," Psychonomic Bulletin 83 Review 1(2), pp. 202-238, 1994.

6. R. K. White and J. S. Brush, "Analyzing resolution requirements of automated target recognition systems," in Signal Processing, Sensor Fusion, and Target Recognition, V. Libby and I. Kadar, eds., 1699, pp. 267-277, SPIE, July 1992.

7. G. O'Brien, Encyclopedia of Optical Engineering, vol. 1, ch. Automatic Target Recognition, pp. 114-120. Marcel Dekker, 2003.

8. S. Frintrop, E. Rome, A. Nchter, and H. Surmann, "An attentive, multi-modal laser "eye"," in Computer Vision Systems: Third International Conference, Lecture Notes in Computer Science 2626, pp. 202-211, ICVS, Springer Berlin / Heidelberg, Apr. 2003.

9. W. M. Brown and C. W. Swonger, "A prospectus for automatic target recognition," IEEE Transactions on Aerospace and Electronic Systems 25, pp. 401-410, May 1989.

10. Q. Li, M. Zhou, and J. Liu, "Multi-resolution mesh based 3d object recognition," in Computer Vision Beyond the Visible Spectrum: Methods and Applications, pp. 37-43, SPIE, 2000.

11. W. C. Stone, M. Juberts, N. Dagalakis, J. Stone, and J. Gorman, "Performance analysis of next-generation ladar for manufacturing, construction, and mobility," NISTIR 7117, NIST, Building and Fire Research Laboratory National Institute of Standards and Technology Gaithersburg, Maryland 20899, May 2004.

12. P. J. Burt, "Smart sensing within a pyramid vision machine," in Proceedings of the IEEE, 76, pp. 1006-1015, IEEE, Aug. 1988.

13. J. D. Baker and W. M. Wells, III, "Multiresolution statistical object recognition," in ARPA94, 2, pp. 12511256, 1994.

14. W. S. Geisler and J. S. Perry, "A real-time foveated multiresolution system for low-bandwidth video communication," in Human Vision and Electronic Imaging III, B. E. Rogowitz and T. N. Pappas, eds., 3299, pp. 294-305, SPIE, July 1998.

15. H. R. Sheikh, B. L. Evans, and A. C. Bovik, "Real-time foveation techniques for low bit rate video coding," Real-Time Imaging 9(1), pp. 27-40, 2003.

16. D. C. McKee, C. Bandera, S. Ghosal, and P. J. Rauss, "Model-based automatic target recognition using hierarchical foveal machine vision," in Signal Processing, Sensor Fusion, and Target Recognition V, I. Kadar and V. Libby, eds., 2755, pp. 70-79, SPIE, June 1996.

17. Y. Amit, D. Geman, and X. Fan, "A coarse-to-fine strategy for multiclass shape detection," IEEE Transactions on Pattern Analysis and Machine Intelligence 26, pp. 1606-1621, Dec. 2004.

18. M. Montemerlo and S. Thrun, "A multi-resolution pyramid for outdoor robot terrain perception.," in Proceedings of the Nineteenth National Conference on Artificial Intelligence, Sixteenth Conference on Innovative Applications of Artificial Intelligence, D. L. McGuinness and G. Ferguson, eds., pp. 464-469, AAAI, AAAI Press / The MIT Press, July 2004.

19. J. S. Zelek, "Complete real-time path planning during sensor-based discovery," in Intelligent Robots and Systems, 3, pp. 1399-1404, IEEE, Oct. 1998.

20. H. Wechsler, "Hierarchical and modular attention," in Computer Architectures for Machine Perception, pp. 222-231, IEEE, Sept. 1995.

21. P. Sajda, C. Spence, L. Parra, and R. Nishikawa, "Hierarchical, multi-resolution models for object recognition: Applications to mammographic computer-aided diagnosis," in Applied Imagery Pattern Recognition Workshop, pp. 159-165, IEEE, 2000.

22. V. P. Concepcion and H. Wechsler, "Multiresolution attention and associative memory systems for timevarying imagery," in Pattern Recognition, Conference A: Computer Vision 83 Image Processing 1, pp. 840842, SPIE, 1994.

23. K. M. Iftekharuddin and R. P. Malhotra, "Role of multiresolution attention in automated object recognition," in Proceedings of the 2002 International Joint Conference on Neural Networks, 3, pp. 2255-2260, IEEE, 2002. 
24. M. Snorrason, H. Ruda, and A. Caglayan, "Automatic target recognition in laser radar imagery," in Acoustics, Speech, and Signal Processing, 4, pp. 2471-2474, IEEE, 1995.

25. V. P. Concepcion and H. Wechsler, "Zoom lens for automatic target recognition (atr)," in Signal Processing, Sensor Fusion, and Target Recognition, V. Libby and I. Kadar, eds., 1699, pp. 208-217, SPIE, July 1992.

26. H. D. Tagare, K. Toyama, and J. G. Wang, "A maximum-likelihood strategy for directing attention during visual search," IEEE Transactions on Pattern Analysis and Machine Intelligence 23, pp. 490-500, May 2001.

27. L. Itti, C. Koch, and E. Niebur, "A model of saliency-based visual attention for rapid scene analysis," IEEE Transactions on Pattern Analysis and Machine Intelligence 20, pp. 1254-1259, Nov. 1998.

28. L. Itti and C. Koch, "A saliency-based search mechanism for overt and covert shifts of visual attention," Vision Res 40(10-12), pp. 1489-1506, 2000.

29. C. M. Privitera and L. W. Stark, "Algorithms for defining visual regions-of-interest: comparison witheye fixations," IEEE Transactions on Pattern Analysis and Machine Intelligence 22, pp. 970-982, Sept. 2000.

30. U. Rutishauser, D. Walther, C. Koch, and P. Perona, "Is bottom-up attention useful for object recognition?," in Proceedings of the 2004 IEEE Computer Society Conference on Computer Vision and Pattern Recognition, 2, pp. 37-44, IEEE, June 2004.

31. D. Parkhursta, K. Law, and E. Niebur, "Modeling the role of salience in the allocation of overt visual attention," Vision Research 42, pp. 107-123, Jan. 2002.

32. D. Walther, U. Rutishauser, C. Koch, , and P. Perona, "On the usefulness of attention for object recognition," in 2nd Workshop on Attention and Performance in Computational Vision, pp. 96-103, European Computer Vision Confererence (ECCV04), May 2004.

33. S. Frintrop, A. Nuchter, H. Surmann, and J. Hertzberg, "Saliency-based object recognition in 3d data," in Proceeding of 2004 IEEE/RSJ International Conference onIntelligent Robots and Systems, 3, pp. 2167-2172, IEEE, Sept. 2004.

34. V. Navalpakkam and L. Itti, "Sharing resources: Buy attention, get recognition," in Proc. International Workshop on Attention and Performance in Computer Vision (WAPCV'03), Jul 2003.

35. L. Itti, "Visual salience." Scholarpedia on the World Wide Web, Sept. 2007.

36. C. Koch and S. Ullman, "Shifts in selective visual attention: towards the underlying neural circuitry," Human Neurobiology 4, pp. 219-227, 1985.

37. F. Moosmann, D. Larlus, and F. Jurie, "Learning saliency maps for object categorization," in $E C C V$ International Workshop on The Representation and Use of Prior Knowledge in Vision, 2006.

38. N. Ouerhani, R. von Wartburg, H. Hugli, and R. Muri, "Empirical validation of the saliency-based model of visual attention," ELVCIA 3(1), pp. 13-24, 2004.

39. J. J. Bonaiuto and L. Itti, "Combining attention and recognition for rapid scene analysis," in Proc. IEEECVPR Workshop on Attention and Performance in Computer Vision (WAPCV'05), San Diego, California, pp. 1-6, June 2005. 